\title{
Impacto de variáveis antropométricas sobre a insatisfação corporal e o comportamento alimentar em jovens atletas
}

\author{
Impact of anthropometric variables on body dissatisfaction \\ and eating behavior in young athletes
}

Leonardo de Sousa Fortes'1, Sebastião de Sousa Almeida², Maria Elisa Caputo Ferreira ${ }^{3}$

\section{RESUMO}

Objetivo: Identificar a influência de variáveis antropométricas sobre a insatisfação corporal (IC) e o comportamento alimentar inadequado (CAI) em atletas adolescentes competitivos. Métodos: Participaram 580 jovens (464 meninos e 116 meninas) com idades entre 10 e 19 anos, de ambos os sexos. Aplicou-se o Body Shape Questionnaire para mensurar a IC. Utilizou-se o Eating Attitudes Test para avaliar CAI. Foram aferidas dobras cutâneas para estimar o percentual de gordura (\%G). Mensuraram-se peso e estatura para calcular o IMC. Resultados: Os resultados evidenciaram que a IC no sexo feminino foi modulada apenas pelo \%G, ao contrário do sexo masculino, em que IMC e \%G, juntos, explicaram parte de sua variância $(p<0,05)$. O CAI no sexo masculino foi pouco influenciado pelo \%G. Conclusão: Pode-se

\section{Palavras-chave}

Comportamento alimentar, imagem corporal, atletas.

\section{Keywords}

Eating behavior, body image, athletes. concluir que o \%G foi a única variável que influenciou a IC em ambos os sexos. Ademais, os CAl em jovens atletas parecem não ser influenciados pelas características antropométricas mensuradas neste estudo.

\section{ABSTRACT}

Objective: To identify the influence of anthropometric variables on the body dissatisfaction (BD) and the inappropriate eating behavior (IEB) in competitive teenage athletes. Methods: 580 young (464 boys and 116 girls) between the ages of 10 and 19 years of both sexes was participed. The Body Shape Questionnaire was applied to measure the BD. The Eating Attitudes Test was used to evaluate IEB. Skinfolds were measured to estimate the percentage of body fat (\%F). Weight and height were measured to calculate the BMI. Results: The results showed that the BD in female gender was modulated only by \%F, unlike the males, in which $\mathrm{BMI}$ and $\% \mathrm{~F}$, together, explained part of its variance $(\mathrm{p}<0.05)$. The IEB in male gender was little influenced by \%F. Conclusion: It can be concluded that the \%F was the only variable that influenced the BD in both genders. Furthermore, the IEB on young athletes doesn't seem to be influenced by anthropometric characteristics measured this study.

1 Universidade Federal de Juiz de Fora (UFJF), Faculdade de Educação Física e Desportos; Conselho Nacional de Desenvolvimento Científico e Tecnológico (CNPq), Grupo de Pesquisa "Corpo e Diversidade Humana". 


\section{INTRODUÇÃO}

Existem alguns indicadores antropométricos de sobrepeso/ obesidade. O índice de massa corporal (IMC) é um deles, sendo amplamente utilizado na avaliação do estado nutricional'. A Organização Mundial da Saúde (OMS)² recomenda a utilização do IMC para diagnosticar tanto a obesidade quanto a desnutrição em adolescentes. O IMC é bastante utilizado por causa do baixo custo, da simplicidade para a realização das medidas e da alta reprodutibilidade 3 . Altos valores de IMC têm sido fortemente associados a insatisfação corporal' e ao comportamento alimentar inadequado em adolescentes ${ }^{4,5}$, porém ainda não foi comprovado se o IMC possui influência sobre essas variáveis em atletas 6 .

Além do IMC, a adiposidade corporal também pode ser utilizada como ponto de corte de sobrepeso/obesidade4. Para isso, são necessárias mensurações em dobras cutâneas para estimar o percentual de gordura ${ }^{7}$. Pesquisas sugerem que a gordura corporal influencia tanto a insatisfação corporal quanto o comportamento alimentar em atletas ${ }^{8,9}$. Todavia, não existem evidências a respeito dessa possível influência em jovens atletas brasileiros.

A adiposidade corporal e o IMC sofrem variações principalmente no período de maior crescimento físico, ocorrido durante a puberdade ${ }^{10}$. Em adição, a ingestão de alimentos e a prática regular de exercícios físicos também podem interferir nessas variações supracitadas ${ }^{11}$. Essas modificações morfológicas podem influenciar no comportamento alimentar e na imagem corporal de adolescentes'.

A imagem corporal é definida como a imagem mental a respeito do corpo ${ }^{12}$. Um dos subcomponentes da dimensão atitudinal da imagem corporal que vale a pena ser investigado é a insatisfação corporal, que diz respeito à depreciação que o indivíduo tem com sua aparência física'. Segundo Bonci et al. ${ }^{8}$, a insatisfação com o corpo é um dos fatores predisponentes para o comportamento alimentar inadequado, que, por sua vez, pode desencadear os transtornos alimentares (TA). Parece que tanto a insatisfação corporal quanto o comportamento alimentar anormal manifestamse com frequências diferentes entre os sexos. Estudos têm apresentado resultados que mostram maior prevalência de transtornos alimentares entre indivíduos do sexo feminino ${ }^{4,5}$, recomendando-se avaliar essas variáveis segundo o gênero.

Os TA mais comumente conhecidos são a anorexia nervosa (AN) e a bulimia nervosa (BN). Essas psicopatologias são caracterizadas por medo mórbido de engordar, distorção perceptiva da imagem corporal e uso de métodos patológicos para emagrecimento ${ }^{5}$. São doenças que parecem afetar em maior escalão a população de atletas ${ }^{6,8}$. Evidências indicam que aproximadamente 15\% dos atletas podem desenvolver algum tipo de transtorno alimentar, no entanto menos de 5\% dessa população possuem diagnóstico clínico para essa psicopatologia9. Desse modo, faz-se necessário o desenvolvimento de investigações com o propósito de identificar possíveis fatores que possam ser considerados desencadeadores dos TA. Todavia, ainda são escassos os estudos que tenham avaliado a influência de características antropométricas sobre os comportamentos alimentares e a insatisfação corporal em jovens atletas. Estima-se que esta seja a primeira pesquisa no Brasil a estudar tais influências nessa população. Diante do pressuposto, o objetivo do presente estudo foi identificar a influência de variáveis antropométricas sobre a insatisfação corporal e o comportamento alimentar inadequado em atletas adolescentes.

\section{MÉTODOS}

Trata-se de um estudo transversal, com característica de associação. A priori, as federações de várias modalidades esportivas e alguns clubes localizados nos quatro estados brasileiros pertencentes à Região Sudeste foram contatados e convidados por telefone ou e-mail a participar da pesquisa. No entanto, obtiveram-se respostas somente nos estados do Rio de Janeiro (RJ) e Minas Gerais (MG). Sendo assim, a população da pesquisa representou atletas adolescentes com idades entre 10 e 19 anos, de ambos os sexos, residentes nos estados do Rio de Janeiro/RJ e Minas Gerais/MG no Brasil. Segundo o Comitê Olímpico Brasileiro, no ano de 2011, essa população era na ordem de 26.467 indivíduos. Realizou-se o cálculo amostral, considerando prevalência de $20 \%$ para a insatisfação corporal ${ }^{6,13} \mathrm{com} 95 \%$ de confiança, $4 \%$ de erro amostral e efeito de desenho de 1,4, totalizando 379 jovens. Todavia, por recomendações de Schaal et al. ${ }^{9}$, decidiu-se coletar o máximo de dados possível, além do tamanho amostral calculado. Somente foram incluídos na pesquisa sujeitos que apresentassem rotina de treinamento físico sistematizado, com frequência e duração mínima de cinco vezes na semana e $1 \mathrm{~h} /$ dia, respectivamente. Acrescentando, os atletas deveriam ter participado de competições oficiais. Desse modo, foram incluídos no estudo 620 atletas de ambos os sexos com tempo médio de treinamento de $3,19( \pm 1,08)$ anos de acordo com seu esporte praticado, porém 40 desses sujeitos foram excluídos da amostra por não responderem aos questionários em sua totalidade e/ou não participarem da aferição de medidas antropométricas, totalizando uma amostra final de 580 esportistas, conforme ilustrado na tabela 1. Para seleção da amostra, foi utilizado o método de amostragem casual simples'. Este estudo somente foi desenvolvido após receber o número do parecer do comitê de ética e pesquisa em seres humanos da Universidade Federal de Juiz de Fora.

\section{Instrumentos}

\section{Body Shape Questionnaire (BSQ)}

A insatisfação corporal foi avaliada pelo BSQ. Esse instrumento possui 34 questões em escala likert, variando de $1=$ nunca 
até 6 = sempre. Essas questões avaliam a insatisfação com o peso e a aparência física. A versão utilizada foi validada para adolescentes brasileiros ${ }^{12}$. Calculou-se o alfa de Cronbach para a presente amostra, sendo encontrados valores de 0,91 para as meninas e 0,92 para os meninos. A pontuação final é resultado da soma dos escores de cada item do questionário. Quanto maior a pontuação, maior é a depreciação com o peso e a aparência corporal.

\section{Eating Attitudes Test (EAT-26)}

Para avaliar o comportamento alimentar inadequado, foi utilizado o questionário EAT-26, composto por 26 questões distribuídas em diferentes aspectos: dieta, bulimia nervosa e preocupação com comida e autocontrole oral. O EAT-26 aplicado em atletas adolescentes do sexo feminino foi validado por Bighetti et al. ${ }^{14}$. Para esportistas do sexo masculino, foi utilizado o EAT-26 validado para adolescentes brasileiros ${ }^{15}$. Para amostra do presente estudo, o alfa de Cronbach foi calculado, identificando-se valores de 0,89 e 0,92 para meninas e meninos, respectivamente. A pontuação do EAT-26 é feita pela soma dos seus itens. Escore igual ou maior que 20 representa indivíduos com comportamento alimentar de risco para TA. Existem seis opções de resposta que variam de 0 a 3 pontos (sempre $=3$, muitas vezes $=2$, frequentemente $=1$, poucas vezes $=0$, quase nunca $=0$ e nunca $=0$ ), e a questão 25 apresenta pontuação inversa (sempre $=0$, muitas vezes $=$ 0 , frequentemente $=0$, poucas vezes $=1$, quase nunca $=2$ e nunca $=3)$.

\section{Antropometria}

O percentual de gordura foi estimado pelo método duplamente indireto, mensurando-se as dobras cutâneas tricipital e subescapular. Utilizou-se o protocolo de Slaughter et al. ${ }^{7}$ para executar tal estimativa. Essas medidas foram realizadas de forma rotacional e coletadas três vezes, considerando-se a média dos valores.

A massa corporal foi mensurada por uma balança digital portátil com precisão de $100 \mathrm{~g}$ e capacidade máxima de $200 \mathrm{~kg}$. Utilizou-se estadiômetro portátil com precisão de 0,1 cm e altura máxima de 2,20 m para mensurar a estatura.

Para todas as medições antropométricas, utilizaram-se as padronizações da ISAK ${ }^{16}$. Ademais, realizou-se o cálculo do erro técnico de medida proposto por Silva et al. ${ }^{17}$, excluindo dados com variância maior que $10 \%$.

Determinou-se a adiposidade corporal pelo percentual de gordura. A classificação dessa variável foi atribuída pelos pontos de corte estabelecidos por Lohman ${ }^{18}$, que levam em consideração o sexo do sujeito (Feminino: $<11 \%=$ muito bai$\mathrm{xo},>12 \%$ e $<15 \%=$ baixo, $>16 \%$ e $<25 \%=$ normal, $>26 \%$ e $<30 \%=$ moderadamente alto, $>31 \%$ e $<35 \%=$ alto, $>35 \%$ $=$ muito alto; Masculino: $<6 \%=$ muito baixo, $>7 \% \mathrm{e}<12 \%=$ baixo, $>13 \%$ e $<18 \%=$ normal, $>19 \%$ e $<25 \%=$ moderadamente alto, $>26 \%$ e $<32 \%=$ alto, $>32 \%=$ muito alto).
O índice de massa corporal [IMC = massa corporal $(\mathrm{kg}) /$ estatura $\left(\mathrm{m}^{2}\right)$ ] foi usado como indicador de estado nutricional. A classificação do IMC obedeceu aos critérios da OMS², que propõe a classificação em baixo peso, peso normal, sobrepeso e obeso, de acordo com percentis $(5,85$ e 95) em função da idade cronológica.

\section{Procedimentos}

Os treinadores/diretores de 14 clubes foram convidados a participar da pesquisa, sendo informados sobre objetivos e procedimentos do estudo. No entanto, somente 10 deles concordaram em liberar os atletas para participação nas coletas. Em seguida, foram realizadas reuniões com cada uma das equipes a fim de explicar os objetivos e procedimentos necessários para inclusão dos atletas no estudo. Foi entregue o termo de consentimento livre e esclarecido (TCLE) aos adolescentes, pedindo-lhes que devolvessem devidamente assinado pelos responsáveis na semana seguinte, em caso de assentimento de sua participação voluntária.

As coletas de dados foram realizadas por apenas um pesquisador. No primeiro encontro, foram aplicados os instrumentos BSQ e EAT-26 de forma coletiva. Entretanto, durante essa etapa não se permitiu que os atletas se comunicassem. Os questionários foram entregues aos atletas que receberam a mesma orientação verbal. Eventuais dúvidas foram esclarecidas pelo responsável pela aplicação desses instrumentos. Não houve limite de tempo para preenchê-los.

No segundo encontro, os atletas foram conduzidos para aferição das variáveis antropométricas. A entrada dos atletas na sala de avaliação foi individualizada, permitindo-se outro sujeito adentrar no ambiente somente após seu colega de equipe retirar-se do recinto. Todos esses procedimentos foram realizados entre os meses de março e junho de 2011.

\section{Análise estatística}

Para avaliar a influência de variáveis antropométricas sobre a insatisfação com o corpo e o comportamento alimentar, adotou-se como parâmetro a regressão linear múltipla stepwise. Antes da realização dessa análise, aplicou-se o teste de normalidade Kolmogorov-Smirnov nas variáveis dependentes (BSQ e EAT-26). Como não foi identificada violação na distribuição dos dados, procedeu-se à regressão linear múltipla. Para essa análise, o IMC, utilizado no primeiro bloco, e o percentual de gordura, inserido no segundo bloco, foram utilizados como variáveis explicativas. Essas duas variáveis explicativas do modelo de regressão demonstraram colinearidade entre si tanto no sexo feminino $(r=0,68$; $p=0,001)$ quanto no masculino $(r=0,56 ; p=0,001)$, avaliado por meio da correlação de Pearson. Ademais, foi realizada regressão logística binária para avaliar riscos do estado nutricional e da adiposidade corporal na predisposição sobre a insatisfação corporal e o comportamento alimentar inadequado. Para esse teste, as classificações do BSQ "leve 
insatisfação", "moderada insatisfação" e "grave insatisfação" foram agrupadas em "insatisfeitos". As classificações de adiposidade corporal "muito baixo" e baixo" foram designadas como "baixo", e as classificações "moderadamente alto", "alto" e "muito alto" foram agrupadas em "alto". Os testes estatísticos foram realizados no software SPSS versão 17.0, incutindo nível de significância de 5\%.

\section{RESULTADOS}

Evidenciaram-se médias de $14,54( \pm 1,86)$ e $15,05( \pm 2,02)$ anos para a idade em atletas do sexo feminino e masculino, respectivamente. Adicionando, os achados indicaram que $80,2 \%$ das atletas eram de etnia branca, $16,4 \%$ eram negras e $3,4 \%$ se declararam de etnia amarela. No sexo masculino, $62,3 \%$ dos atletas eram de etnia branca, 30,4\% se declararam negros e 7,3\% eram amarelos.

Dos 580 atletas avaliados no presente estudo, 249 eram provenientes do estado do Rio de Janeiro (47 meninas e 202 meninos) e 331, do estado de Minas Gerais (69 meninas e 262 meninos).

A tabela 2 demonstra que a insatisfação corporal no sexo feminino foi modulada apenas pelo percentual de gordura, ao contrário do sexo masculino, em que o IMC e o percentual de gordura, juntos, explicaram parte da variância da insatisfação corporal. O comportamento alimentar inadequado no sexo masculino foi pouco influenciado pelo percentual de gordura (Tabela 3). No sexo feminino, tanto o percentual de gordura quanto o IMC não explicaram a variância do comportamento alimentar inadequado, como pode ser observado na tabela 3.

Tabela 1. Distribuição de frequência dos atletas segundo sexo e modalidade esportiva

\begin{tabular}{lcccc}
\hline Modalidade & \multicolumn{2}{c}{ Feminino } & \multicolumn{2}{c}{ Masculino } \\
\hline Atletismo & FA (n) & FR (\%) & FA (n) & FR (\%) \\
Basquetebol & - & - & 10 & 2,2 \\
Esgrima & 16 & 13,8 & 56 & 12,1 \\
Futebol & - & - & 8 & 1,7 \\
Ginástica artística & 14 & - & 271 & 58,4 \\
Handebol & 20 & 12,1 & - & - \\
Judô & - & - & 16 & 3,4 \\
Natação & 22 & 19,0 & 14 & 3,0 \\
Polo aquático & - & - & 42 & 9,1 \\
Saltos & 5 & 4,3 & 16 & 3,4 \\
ornamentais & & & 5 & 1,1 \\
Tae-kwon-do & 5 & 4,3 & 6 & 1,3 \\
Triathlon & - & - & 14 & 3,0 \\
Voleibol & 16 & $100 \%$ & 464 & 1,3 \\
Total & 116 & & & $100 \%$ \\
\hline FAfrequenca & & & 6 &
\end{tabular}

FA: frequência absoluta; FR: Frequência relativa.
Tabela 2. Regressão linear múltipla utilizando o IMC e percentual de gordura como variáveis explicativas sobre a variância da insatisfação corporal de atletas adolescentes competitivos divididos por sexo

\begin{tabular}{cccccccc}
\hline Sexo & Variável & Bloco & $B$ & $R$ & $R^{2}$ & $\mathbb{R}^{2 *}$ & $p$ valor \\
\hline $\mathrm{F}$ & $\mathrm{IMC}$ & 1 & 0,11 & 0,35 & 0,12 & 0,11 & $p \leq 0,39$ \\
& $\% \mathrm{G}$ & 2 & 0,17 & 0,44 & 0,19 & 0,18 & $p \leq 0,01$ \\
& $\mathrm{IMC} \% \mathrm{G}$ & 3 & 3,56 & 0,44 & 0,19 & 0,18 & $p \leq 0,01$ \\
$\mathrm{M}$ & $\mathrm{IMC}$ & 1 & 0,16 & 0,37 & 0,14 & 0,14 & $p \leq 0,01$ \\
& $\% \mathrm{G}$ & 2 & 0,15 & 0,36 & 0,13 & 0,13 & $p \leq 0,01$ \\
& $\mathrm{IMC} \% \mathrm{G}$ & 3 & 3,22 & 0,42 & 0,17 & 0,17 & $p \leq 0,01$ \\
\hline
\end{tabular}

F:feminino; $M$ : masculino; $R^{2 *}: R^{2}$ ajustado; $I M C * G$ : interação entre índice de massa corporal e percentual de gordura.

Tabela 3. Regressão linear múltipla utilizando o IMC e percentual de gordura como variáveis explicativas sobre a variância do comportamento alimentar inadequado de atletas adolescentes competitivos divididos por sexo

\begin{tabular}{lccccccc}
\hline Sexo & Variável & Bloco & $\boldsymbol{B}$ & $\mathbf{R}$ & $\mathbf{R}^{2}$ & $\mathbf{R}^{2 *}$ & $\mathrm{p}$ valor \\
\hline $\mathrm{F}$ & $\mathrm{IMC}$ & 1 & 0,08 & 0,15 & 0,02 & 0,02 & $\mathrm{p} \leq 0,09$ \\
& $\% \mathrm{G}$ & 2 & 0,09 & 0,18 & 0,03 & 0,02 & $\mathrm{p} \leq 0,06$ \\
& $\mathrm{IMC} \% \mathrm{G}$ & 3 & 1,16 & 0,19 & 0,03 & 0,02 & $\mathrm{p} \leq 0,14$ \\
$\mathrm{M}$ & $\mathrm{IMC}$ & 1 & 0,03 & 0,06 & 0,004 & 0,001 & $\mathrm{p} \leq 0,2$ \\
& $\% \mathrm{G}$ & 2 & 0,12 & 0,16 & 0,03 & 0,02 & $\mathrm{p} \leq 0,01$ \\
& $\mathrm{IMC} \% \mathrm{G}$ & 3 & 1,89 & 0,17 & 0,03 & 0,02 & $\mathrm{p} \leq 0,01$ \\
\hline
\end{tabular}

F: feminino; M: masculino; $R^{2 *}: R^{2}$ ajustado; $I M C * \% G$ : interação entre índice de massa corporal e percentual de gordura.

Ademais, a insatisfação corporal esteve associada ao estado nutricional e à adiposidade corporal em ambos os sexos (Tabela 4). Quando se ajustou o modelo para todas as variáveis, o estado nutricional manteve-se associado com a insatisfação corporal nos dois sexos. Entretanto, a adiposidade corporal manteve-se relacionada com a insatisfação corporal somente no sexo feminino.

Tabela 4. Razão de chances para insatisfação com a imagem corporal (categoria de referência: satisfeitos) em atletas adolescentes divididos por sexo segundo indicadores antropométricos

\begin{tabular}{|c|c|c|c|c|c|c|}
\hline Sexo & Variável & Classificação & $O R$ & IC (95\%) & $O R^{*}$ & IC (95\%) \\
\hline \multirow[t]{7}{*}{$\mathrm{F}$} & $\mathrm{EN}^{\mathrm{a}, \mathrm{b}}$ & Baixo peso & 6,09 & $0,60-60,92$ & 7,39 & $0,72-75,22$ \\
\hline & & Peso normal & 1,00 & & 1,00 & \\
\hline & & Sobrepeso & 2,84 & $0,84-9,66$ & 1,37 & $0,35-5,38$ \\
\hline & & Obeso & 3,28 & $0,50-21,99$ & 1,15 & $0,1-13,15$ \\
\hline & $\% G^{a, b}$ & Baixo & 0,27 & $0,03-2,28$ & 0,31 & $0,36-2,62$ \\
\hline & & Normal & 1,00 & & 1,00 & \\
\hline & & Alto & 3,94 & $1,63-9,50$ & 3,44 & $1,29-9,17$ \\
\hline \multirow[t]{7}{*}{ M } & $\mathrm{EN}^{\mathrm{a}, \mathrm{b}}$ & Baixo peso & 0,10 & $0,05-4,76$ & 0,10 & $0,03-14,12$ \\
\hline & & Peso normal & 1,00 & & 1,00 & \\
\hline & & Sobrepeso & 6,88 & $3,47-13,63$ & 5,85 & $2,86-11,94$ \\
\hline & & Obeso & 9,41 & $3,94-22,49$ & 7,67 & $3,11-18,90$ \\
\hline & $\% G^{a}$ & Baixo & 0,48 & $0,16-1,47$ & 0,57 & $0,18-1,78$ \\
\hline & & Normal & 1,00 & & 1,00 & \\
\hline & & Alto & 2,38 & $1,32-4,28$ & 1,36 & $0,71-2,59$ \\
\hline
\end{tabular}

F: feminino; M: masculino; EN: estado nutricional; \%G: adiposidade corporal; OR: razão de chances (odds ratio); *: OR ajustada para todas as variáveis; IC: intervalo de confiança.

a: $p<0,05$ para modelo de regressão logística simples; $b: p<0,05$ para modelo de regressão logística ajustada. 
No sexo feminino, os modelos de regressão não indicaram associação com o comportamento alimentar inadequado (Tabela 5). No sexo masculino, somente o estado nutricional apresentou associação com o comportamento alimentar nos dois modelos.

Tabela 5. Razão de chances para comportamento alimentar inadequado (categoria de referência: negativo) em atletas adolescentes divididos por sexo segundo indicadores antropométricos

\begin{tabular}{|c|c|c|c|c|c|c|}
\hline Sexo & Variável & Classificação & OR & IC(95\%) & $O R^{*}$ & IC (95\%) \\
\hline \multirow[t]{7}{*}{$\mathrm{F}$} & EN & Baixo peso & 1,57 & $0,15-16,00$ & 1,51 & $0,15-15,67$ \\
\hline & & Pesonormal & 1,00 & & 1,00 & \\
\hline & & Sobrepeso & 0,94 & $0,19-4,68$ & 0,89 & $0,16-5,08$ \\
\hline & & Obeso & 2,35 & $0,20-27,45$ & 2,22 & $0,16-31,15$ \\
\hline & $\% G$ & Baixo & 0,55 & $0,06-4,79$ & 0,57 & $0,07-4,92$ \\
\hline & & Normal & 1,00 & & 1,00 & \\
\hline & & Alto & 1,06 & $0,37-3,08$ & 1,02 & $0,30-3,48$ \\
\hline \multirow[t]{7}{*}{ M } & $\mathrm{EN}^{\mathrm{a}, \mathrm{b}}$ & Baixo peso & 1,14 & $0,32-4,04$ & 1,38 & $0,37-5,10$ \\
\hline & & Peso normal & 1,00 & & 1,00 & \\
\hline & & Sobrepeso & 1,19 & $0,50-2,81$ & 1,15 & $0,47-2,79$ \\
\hline & & Obeso & 2,66 & $1,05-6,75$ & 2,52 & $0,96-6,60$ \\
\hline & $\% G$ & Baixo & 0,44 & $0,18-1,12$ & 0,44 & $0,17-1,12$ \\
\hline & & Normal & 1,00 & & 1,00 & \\
\hline & & Alto & 0,97 & $0,56-1,68$ & 0,88 & $0,49-1,59$ \\
\hline
\end{tabular}

F: feminino; M: masculino; EN: estado nutricional; \%G: adiposidade corporal; OR: razão de chances (odds ratio);

*: OR ajustada para todas as variáveis; IC: intervalo de confiança.

a: $p<0,05$ para modelo de regressão logística simples, ${ }^{b}: p<0,05$ para modelo de regressão logística ajustada.

\section{DISCUSSÃO}

Os achados do presente estudo mostraram que o IMC modulou a insatisfação corporal somente no sexo masculino, porém o estado nutricional apresentou associação com a insatisfação corporal no modelo de regressão logística ajustada em ambos os sexos. Meninas com baixo peso e sobrepeso foram apontadas com maior tendência (7,39 e 1,37, respectivamente) de serem insatisfeitas com a aparência física, comparadas às atletas classificadas em peso normal. Os achados do sexo feminino corroboram tendências expostas pela literatura de que adolescentes com sobrepeso/obesidade são mais insatisfeitas com o peso e a aparência corporal ${ }^{1,5}$. Entretanto, encontrar uma razão de 7,39 vezes mais chances de insatisfação corporal em atletas com estado nutricional baixo peso não era esperado, visto que geralmente meninas almejam reduzir peso e apresentar a magreza como ideal estético ${ }^{4,19}$. Esses resultados corroboram o estudo de De Bruin et al..20, que identificaram que atletas femininas, mesmo com baixo peso corporal, estavam insatisfeitas com o corpo e ainda desejavam reduzir peso. Portanto, os resultados no sexo feminino podem ser reflexo das características de parte da amostra que provinha de modalidades de cunho estético (ginástica artística, nado sincronizado e saltos ornamentais), pois pesquisas mostram que nesses esportes, independentemente das características morfológicas, atletas costumam apresentar insatisfação com o corpo ${ }^{20-22}$. Já no sexo masculino, sobrepeso e obesidade foram preditores de insatisfação com o corpo, o que já era esperado. Apesar da escassez de estudos de associação, os achados desta pesquisa parecem ser semelhantes a resultados de estudos com escolares da mesma faixa etária4. Talvez, o estado nutricional manifeste sentimentos afetivos semelhantes nos dois sexos na população de jovens atletas, já que o excesso de peso é considerado aspecto depreciativo na cultura ocidental ${ }^{5}$.

A presente pesquisa evidenciou que o percentual de gordura influenciou significativamente a variância da insatisfação corporal em ambos os sexos, sendo esta modulada em 19\% nas meninas e 13\% nos meninos. A adiposidade corporal esteve associada apenas no sexo feminino no modelo de regressão logística. Meninas com alto percentual de gordura apresentaram 3,44 vezes mais chances de insatisfação corporal comparadas a atletas com adiposidade corporal normal. Em atletas, a adiposidade corporal, além de influenciar a insatisfação corporal, parece ser preditora dela no sexo feminino, pois a quantidade de gordura é inversamente proporcional ao rendimento esportivo em diversas modalidades ${ }^{13,19}$. Por isso, algumas atletas, juntamente com seus treinadores, acreditam que um baixo percentual adiposo é característica fundamental para a otimização da performance. No entanto, percentual de gordura muito baixo pode predispor essas atletas a problemas fisiológicos como a amenorreia e psicológicos como a AN23. Por outro lado, a gordura corporal não foi preditora de insatisfação corporal no sexo masculino. Meninos parecem se incomodar menos com aspectos morfológicos quando comparados a meninas ${ }^{24}$, independentemente de serem atletas ou não'. Atletas do sexo masculino provavelmente estão mais preocupados com os resultados esportivos do que com sua composição corporal13,19.

Os resultados do presente estudo mostraram que o IMC e o percentual de gordura não influenciaram na variância do EAT-26 no sexo feminino. Por conseguinte, estado nutricional e adiposidade corporal não foram preditores significativos de hábitos alimentares não saudáveis nesse sexo. Os achados da pesquisa de Haase ${ }^{25}$ são contraditórios aos do presente estudo, já que essa autora, avaliando atletas de diversas modalidades, encontrou pontuações significativamente superiores no EAT-26 em indivíduos com sobrepeso. De Bruin et al. ${ }^{26}$ mostraram resultados semelhantes aos de Haase $^{25}$, pois identificaram que o IMC influenciou a frequência de comportamentos patológicos para o controle de peso em atletas holandesas. Parece que meninas preocupam-se com hábitos alimentares mesmo apresentando peso e gordura corporal dentro dos padrões de normalidade ${ }^{5,21}$, o que pode explicar, de certo modo, os achados do presente estudo. Ademais, as características das modalidades avaliadas 
na presente pesquisa são diferentes das dos estudos supracitados, devendo-se, então, ter cautela nas comparações e interpretações.

Nos meninos, os resultados apontaram que apenas o percentual de gordura influenciou o comportamento alimentar inadequado (3\%) e somente a adiposidade corporal manteve-se associada com o fator positivo do EAT-26 no modelo de regressão logística. Esta última análise evidenciou que atletas com sobrepeso e obesidade, respectivamente, apresentaram 5,85 e 7,67 vezes mais chances de alimentação desordenada do que atletas eutróficos. Nesse público, as pesquisas realizadas geralmente associaram o comportamento alimentar inadequado a aspectos psicológicos como perfeccionismo ${ }^{27}$, ansiedade competitiva ${ }^{8}$, dependência de substâncias ${ }^{9}$, autoestima e depressão ${ }^{27}$. Não foram encontrados estudos associando ou averiguando a influência de indicadores antropométricos sobre o comportamento alimentar inadequado em atletas do sexo masculino. Entretanto, diferentemente dos resultados no sexo feminino, parece que o excesso de peso e a elevada adiposidade corporal podem ser considerados fatores de risco para o comportamento alimentar anormal nos atletas masculinos ${ }^{28}$.

Apesar de a presente pesquisa identificar achados inéditos e relevantes, ela apresentou limitações. Uma delas foi utilizar questionários como instrumentos de avaliação. Pesquisadores afirmam que participantes podem não responder com fidedignidade a instrumentos autoaplicáveis ${ }^{19,21,28}$. Portanto, os resultados podem não refletir a realidade do contexto avaliado, visto que o resultado final é fruto de respostas subjetivas. Outra limitação foi utilizar um método duplamente indireto para estimar a gordura corporal dos atletas. Entretanto, ressalta-se a dificuldade de acesso a equipamentos sofisticados, além do alto dispêndio financeiro na utilização desses aparelhos.

\section{CONCLUSÃO}

Por fim, pode-se dizer que as atletas abaixo do peso recomendado para a sua faixa etária e os atletas acima do peso para sua idade (sobrepeso/obeso) demonstraram risco aumentado para a insatisfação corporal. Por outro lado, pode-se afirmar que apenas esportistas com acentuada adiposidade corporal apresentaram riscos para a insatisfação corporal no sexo feminino. Além disso, o percentual de gordura foi a única variável que influenciou a insatisfação corporal em ambos os sexos.

Em relação ao comportamento alimentar inadequado, pode-se concluir que somente os atletas considerados obesos obtiveram riscos aumentados para a adoção de condutas alimentares prejudiciais à saúde no sexo masculino. Entre as meninas, não se identificou um bom indicador antropométrico para predizer hábitos alimentares inadequados. Contudo, ressalta-se que os achados da presente investigação se limitam a essa amostra pesquisada.

\section{AGRADECIMENTOS}

Aos atletas participantes deste estudo, pela colaboração, ao Laboratório de Estudos do Corpo da Universidade Federal de Juiz de Fora (UFJF), pela disponibilização dos instrumentais do estudo, e ao Conselho Nacional de Desenvolvimento Científico e Tecnológico (CNPq), pelo financiamento do projeto.

\section{REFERÊNCIAS}

1. Conti MA, Frutuoso MFP, Gambardella AMD. Excesso de peso e insatisfação corporal em adolescentes. Rev Nutr. 2005;18(4):491-7.

2. World Health Organization. Development of a WHO growth reference for school-aged children and adolescents. Bull World Health Organ. 2007:85(9):660-7.

3. Romero A, Slater B, Florindo AA, Latorre MRDO, Cezar C, Silva MV. Determinantes do índice de massa corporal em adolescentes de escolas públicas de Piracicaba, São Paulo. Cienc Saúde Coletiva. 2010;15(1):141-9.

4. Martins CR, Pelegrini A, Matheus SC, Petroski EL. Insatisfação com a imagem corporal e a relação com estado nutricional, adiposidade corporal e sintomas de anorexia e bulimia em adolescentes. Rev Psiquiatr Rio Gde Sul. 2010;32(1):19-23.

5. Scherer FC, Martins CB, Pelegrini A, Matheus SC, Petroski EL. Imagem corporal em adolescentes: associação com a maturação sexual e sintomas de transtornos alimentares. Rev Bras Psiquiatr. 2010;59(3):198-202.

6. Fortes LS, Oliveira, FG, Ferreira MEC. Influência de fatores afetivos, antropométricos e sociodemográficos sobre o comportamento alimentar em jovens atletas. J Bras Psiquiatr. 2012;61(3):148-53.

7. Slaughter MH, Lohman TG, Boileau R, Hoswill CA, Stillman RJ, Yanloan MD, et al. Skinfold equations for estimation of body fatness in children and youth. Hum Biol. 1988;60:709-23.

8. Bonci CM, Bonci LJ, Granger LR, Johnson CL, Malina RM, Milne LW, et al. National athletic trainers' association position statement: preventing, detecting, and managing disordered eating in athletes. J Athl Train. 2008;43(1):80-108.

9. Schaal K, Tafflet M, NassifH, Thibault V, Pichard C, Alcotte M, et al. Psychological balance in high level athletes: gender-based differences and sport-specific patterns. Psychopathology and High Level Sport. 2011;6(5):1-9.

10. Siervogel RM, Demerath EW, Schubert C, Remsberg KE, Chumlea WC, Sun S, et al. Puberty and body composition. Horm Res. 2003;60(1):36-45.

11. Fernandez AC, Mello MT, Tufik S, Castro PM, Fisberg M. Influência do treinamento aeróbio e anaeróbio na massa de gordura corporal de adolescentes obeso. Rev Bras Med Esporte. 2004;10(3):152-8.

12. Conti MA, Cordás TA, Latorre MRDO. Estudo de validade e confiabilidade da versão brasileira do Body Shape Questionnaire (BSQ) para adolescentes. Rev Bras Saúde Mater Infant. 2009;9(3):331-8.

13. Fortes LS, Almeida SS, Ferreira MEC. Processo maturacional, insatisfação corporal e comportamento alimentar inadequado em jovens atletas. Rev Nutr. 2012;25(5):575-86.

14. Bighetti F, Santos CB, Santos JE, Ribeiro RPP. Tradução e avaliação do Eating Attitudes Test em adolescentes do sexo feminino de Ribeirão Preto, São Paulo. J Bras Psiquiatr. 2004:53(6):339-46.

15. Fortes LS, Amaral ACS, Conti MA, Cordás TA, Ferreira MEC. Qualidades psicométricas do Eating Attitudes Test (EAT-26) para a população adolescente masculina brasileira. Psicologia: Reflexão e Crítica (no prelo).

16. Isak - The International Society for Advancement for Kineanthropometry. First printed. Australia: National Library of Australia; 2001.

17. Silva DAS, Pelegrini A, Pires-Neto CS, Vieira MFS, Petroski EL. 0 antropometrista na busca de dados mais confiáveis. Rev Bras Cineantropom Desempenho Hum. 2011;13(1):82-5.

18. Lohman TG. The use of skinfolds to estimate body fatness on children and youth. J Phys Educ Recreational Dance. 1987;58(1):98-103.

19. Krentz EM, Warschburger P. Sports-related correlates of disordered eating in aesthetic sports. Psych Sport Exerc. 2011;44(3):315-21. 
20. De Bruin AP, Oudejans RRD, Bakker FC. Dieting and body image in aesthetic sports: a comparison of dutch female gymnnasts and non-aesthetic sport participants. Psych Sport Exerc. 2007;8(4):507-20

21. Perini TA, Vieira RS, Vigário OS, Oliveira GL, Ornellas JS, Oliveira FP. Transtorno do comportamento alimentar em atletas de elite de nado sincronizado. Rev Bras Med Esporte. 2009;15(1):54-7.

22. Vieira JLL, Amorim HZ, Vieira LF, Amorim AC, Rocha PGM. Distúrbios de atitudes alimentares e distorção da imagem corporal no contexto competitivo da ginástica rítmica. Rev Bras Med Esporte. 2009;15(6):410-4.

23. Torstveit MK, Sundgot-Borgen J. The female athlete triad exists in both elite athletes and controls. Med Sci Sports Exerc. 2005;37(1):1449-59.
24. Petroski EL, Pelegrini A, Glaner MF. Insatisfação corporal em adolescentes rurais e urbanos. Motricidade. 2009;5(4):13-25

25. Haase AM. Weight perception in female athletes: associations with disordered eating correlates and behavior. Eat Behav. 2011;12(1):64-7.

26. De Bruin AP, Woertman L, Bakker FC, Oudejans RRD. Weight-related sport motives and girl's body image, weight control behaviors, and self-esteem. Sex Roles. 2009;60(9):628-42.

27. Filaire E, Rouveix M, Pannafieux C, Ferrand C. Eating attitudes, perfectionism and bodyesteem of elite male judoists and cyclists. J Sports Sci Med. 2007;6(1):50-7.

28. Fortes LS, Miranda VPN, Amaral ACS, Ferreira MEC. Insatisfação corporal de adolescentes atletas e não atletas. J Bras Psiquiatr. 2011;60(4):309-14. 